\begin{tabular}{|c|l|}
\hline Title & Spontaneous decay rates of LA phonons in quasi-isotropic solids \\
\hline Author(s) & Tamura, Shin-ichiro \\
\hline Citation & $\begin{array}{l}\text { Physical Review B, 31/4), 2574-2577 } \\
\text { https://doi.org/40.1103/PhysRevB.31.2574 }\end{array}$ \\
\hline Issue Date & 1985-02-15 \\
\hline Doc URL & http://hdl.handle.net/2115/5968 \\
\hline Rights & Copyright $\odot$ 1985 A merican Physical Society \\
\hline Type & article (author version) \\
\hline File Information & PRB31-4.pdf \\
\hline
\end{tabular}

Instructions for use 


\title{
Spontaneous decay rates of LA phonons in quasi-isotropic solids
}

\author{
Shin-ichiro Tamura \\ Department of Engineering Science, Hokkaido University, \\ Sapporo 060, Japan \\ (Received 13 November 1984)
}

\begin{abstract}
The spontaneous decay rates of LA phonons are calculated in terms of the second- and third-order elastic constants in the isotropic model. It is shown that the dominant decay channel is LA $\rightarrow$ TA + TA but not $\mathrm{LA} \rightarrow \mathrm{LA}+\mathrm{TA}$ and total decay rates at $1-\mathrm{THz}$ frequency range from $10^{5}$ to $10^{6} \mathrm{sec}^{-1}$ for $\mathrm{Si}, \mathrm{Ge}$, $\mathrm{GaAs}, \mathrm{LiF}, \mathrm{NaF}$, and fused silica. These results indicate that the rates of frequency down conversion of the LA phonons assumed currently in the study of the transport properties of high-frequency phonons are incorrect in both their magnitudes and branching ratios.
\end{abstract}

Recently there have been considerable activities in theoretical investigations of high-frequency phonon transport in the presence of elastic scattering and frequency down conversion. ${ }^{1-4}$ At low temperatures, or equivalently in the regime $\hbar \omega \gg k_{B} T$ ( $\omega$ being the angular frequency of a phonon and $T$ the ambient temperature) the down conversion of a phonon is predominantly caused by the splitting into a pair of low-frequency phonons. In the isotropic model employed currently degenerate TA phonons are prohibited to decay through this splitting process owing to the energy and momentum conservation condition, leading to an anomalously long anharmonic lifetime of high-frequency TA phonons. ${ }^{5}$ In contrast, LA phonons can really decay spontaneously via three-phonon processes exhibiting the decay rate proportional to $\omega^{5}$ in the context of the continuum elasticity theory, 5,6 which has recently been verified experimentally. ${ }^{7}$ However, there seem to be still some problems on the magnitude and on the dominant decay channel of the LA phonons. Orbach and Vredevoe ${ }^{5}$ found that the dominant channel is

$$
\mathrm{LA} \rightarrow \mathrm{LA}+\mathrm{TA},
$$

but Klemens ${ }^{6}$ concluded that

$$
\mathrm{LA} \rightarrow \mathrm{TA}+\mathrm{TA}
$$

dominates the process (1) though these two processes yield the decay rates of the same order of magnitudes. More recent works by Markiewicz ${ }^{8}$ and by Tua and Mahan ${ }^{9}$ support, respectively, the former and latter processes as the dominant channel.

In order to settle these disagreements we reevaluate in more detail the spontaneous decay rate of the LA phonons in terms of the second- and third-order elastic constants and give numerical results for several solids. As far as we know, such a calculation has not been made explicitly.

The anharmonic interaction which causes the threephonon processes can be described by the Hamiltonian ${ }^{10,11}$

$$
\begin{aligned}
H^{\prime}=\int d \mathbf{r} & \left(\frac{1}{2}(\beta+\lambda) \zeta_{i i} \zeta_{j k} \zeta_{j k}+(\gamma+\mu) \zeta_{i j} \zeta_{k i} \zeta_{k j}\right. \\
& \left.+\frac{\alpha}{6} \zeta_{i i} \zeta_{j j} \zeta_{k k}+\frac{\beta}{2} \zeta_{i i} \zeta_{j k} \zeta_{k j}+\frac{\gamma}{3} \zeta_{i j} \zeta_{j k} \zeta_{k i}\right),
\end{aligned}
$$

where $\zeta_{i j}=\partial_{j} u_{i}$ ( $\mathbf{u}$ being the displacement vector), $\lambda$ and $\mu$ stand for the Lamé constants, and $\alpha, \beta$, and $\gamma$ are three independent third-order elastic constants in the isotropic model. In Eq. (3) the summation convention over repeated indices is assumed.

To lowest order in $H^{\prime}$ the spontaneous decay rate $\Gamma$ of a phonon of mode $j$ with the wave vector $\mathbf{q}$ is given by

$$
\Gamma(\mathbf{q}, j)=\frac{\pi \hbar}{8 \rho^{3} V \omega(\mathbf{q}, j)} \sum_{\mathbf{q}^{\prime}} \sum_{j^{\prime}, j^{\prime \prime}} \frac{\left|M\left(\mathbf{q}, \mathbf{q}^{\prime}, \mathbf{q}-\mathbf{q}^{\prime} ; j, j^{\prime}, j^{\prime \prime}\right)\right|^{2}}{\omega\left(\mathbf{q}^{\prime}, j^{\prime}\right) \omega\left(\mathbf{q}-\mathbf{q}^{\prime}, j^{\prime \prime}\right)} \delta\left(\omega(\mathbf{q}, j)-\omega\left(\mathbf{q}^{\prime}, j^{\prime}\right)-\omega\left(\mathbf{q}-\mathbf{q}^{\prime}, j^{\prime \prime}\right)\right),
$$

where $\rho$ is the mass density and $V$ is the volume of a sample. The expression for $M$ is as foilows:

$$
\begin{aligned}
M\left(\mathbf{q}, \mathbf{q}^{\prime}, \mathbf{q}^{\prime \prime} ; j, j^{\prime}, j^{\prime \prime}\right)= & (\beta+\lambda)\left[(\mathbf{q} \cdot \mathbf{e})\left(\mathbf{q}^{\prime} \cdot \mathbf{q}^{\prime \prime}\right)\left(\mathbf{e}^{\prime} \cdot \mathbf{e}^{\prime \prime}\right)+\left(\mathbf{q}^{\prime} \cdot \mathbf{e}^{\prime}\right)\left(\mathbf{q}^{\prime \prime} \cdot \mathbf{q}\right)\left(\mathbf{e}^{\prime \prime} \cdot \mathbf{e}\right)+\left(\mathbf{q}^{\prime \prime} \cdot \mathbf{e}^{\prime \prime}\right)\left(\mathbf{q} \cdot \mathbf{q}^{\prime}\right)\left(\mathbf{e} \cdot \mathbf{e}^{\prime}\right)\right] \\
& +(\gamma+\mu)\left\{\left(\mathbf{q} \cdot \mathbf{q}^{\prime}\right)\left[\left(\mathbf{q}^{\prime \prime} \cdot \mathbf{e}^{\prime}\right)\left(\mathbf{e}^{\prime \prime} \cdot \mathbf{e}\right)+\left(\mathbf{q}^{\prime \prime} \cdot \mathbf{e}\right)\left(\mathbf{e}^{\prime} \cdot \mathbf{e}^{\prime \prime}\right)\right]+\left(\mathbf{q}^{\prime} \cdot \mathbf{q}^{\prime \prime}\right)\left[\left(\mathbf{q} \cdot \mathbf{e}^{\prime}\right)\left(\mathbf{e}^{\prime \prime} \cdot \mathbf{e}\right)+\left(\mathbf{q} \cdot \mathbf{e}^{\prime \prime}\right)\left(\mathbf{e} \cdot \mathbf{e}^{\prime}\right)\right]\right. \\
& \left.+\left(\mathbf{q}^{\prime \prime} \cdot \mathbf{q}\right)\left[\left(\mathbf{q}^{\prime} \cdot \mathbf{e}\right)\left(\mathbf{e}^{\prime} \cdot \mathbf{e}^{\prime \prime}\right)+\left(\mathbf{q}^{\prime} \cdot \mathbf{e}^{\prime \prime}\right)\left(\mathbf{e} \cdot \mathbf{e}^{\prime}\right)\right]\right\} \\
& +\alpha(\mathbf{q} \cdot \mathbf{e})\left(\mathbf{q}^{\prime} \cdot \mathbf{e}^{\prime}\right)\left(\mathbf{q}^{\prime \prime} \cdot \mathbf{e}^{\prime \prime}\right)+\beta\left[(\mathbf{q} \cdot \mathbf{e})\left(\mathbf{q}^{\prime} \cdot \mathbf{e}^{\prime \prime}\right)\left(\mathbf{q}^{\prime \prime} \cdot \mathbf{e}^{\prime}\right)+\left(\mathbf{q}^{\prime} \cdot \mathbf{e}^{\prime}\right)\left(\mathbf{q}^{\prime \prime} \cdot \mathbf{e}\right)\left(\mathbf{q} \cdot \mathbf{e}^{\prime \prime}\right)+\left(\mathbf{q}^{\prime \prime} \cdot \mathbf{e}^{\prime \prime}\right)\left(\mathbf{q} \cdot \mathbf{e}^{\prime}\right)\left(\mathbf{q}^{\prime} \cdot \mathbf{e}\right)\right] \\
& +\gamma\left[\left(\mathbf{q} \cdot \mathbf{e}^{\prime}\right)\left(\mathbf{q}^{\prime} \cdot \mathbf{e}^{\prime \prime}\right)\left(\mathbf{q}^{\prime \prime} \cdot \mathbf{e}\right)+\left(\mathbf{q} \cdot \mathbf{e}^{\prime \prime}\right)\left(\mathbf{q}^{\prime} \cdot \mathbf{e}\right)\left(\mathbf{q}^{\prime \prime} \cdot \mathbf{e}^{\prime}\right)\right],
\end{aligned}
$$

where we have employed abbreviated notations $\mathbf{e}^{\prime}=\mathbf{e}\left(\mathbf{q}^{\prime}, j^{\prime}\right)$, etc., for unit polarization vectors e's. Note that the third term of the right-hand side of Eq. (5) vanishes for processes involving the TA phonons.

Now, we apply Eqs. (4) and (5) to the decay of the LA phonons. The energy and momentum conservation limits the allowable splitting processes to (1), (2) and the collinear process $\mathrm{LA} \rightarrow \mathrm{LA}+\mathrm{LA}$. The collinear process yields a finite contribution to the decay rate but is small compared with the former two processes. Furthermore, the presence of the normal dispersion in the crystalline solids which becomes important at high frequencies acts to prohibit the collinear decay. ${ }^{12}$ Hence, we neglect the collinear process in the present calculation. 
For process (1) we find

$$
\begin{aligned}
M\left(\mathbf{q}, \mathbf{q}^{\prime}, \mathbf{q}^{\prime \prime} ; \text { LA }, \text { LA }, \text { TA }\right)= & (2 \beta+4 \gamma+\lambda+3 \mu) \\
& \times \frac{q q^{\prime}}{q^{\prime \prime}}\left[q^{2}-\left(q^{\prime}\right)^{2}\right] \sin \theta \cos \theta,
\end{aligned}
$$

where $q=|\mathbf{q}|$, etc., and $\theta$ is the angle between $\mathbf{q}$ and $\mathbf{q}^{\prime}$. Equation (6) results from the process in which the final TA phonon is polarized in the plane spanned by $\mathbf{q}$ and $\mathbf{q}^{\prime}$. Note that the final state comprising of a TA phonon polarized perpendicular to the same plane does not contribute to the process (1).

For process (2) there exist two possibilities in the polarizations of the final TA phonons; that is, they are simultaneously polarized (a) within or (b) perpendicular to the plane also spanned by $\mathbf{q}$ and $\mathbf{q}^{\prime}$. These final states yield

$$
M_{(\mathrm{a})}\left(\mathbf{q}, \mathbf{q}^{\prime}, \mathbf{q}^{\prime \prime} ; \mathrm{LA}, \mathrm{TA}, \mathrm{TA}\right)=\frac{q q^{\prime}}{q^{\prime \prime}}\left\{[\beta+\lambda+2(\gamma+\mu)]\left(q^{\prime}-q \cos \theta\right)^{2}-(\beta+2 \gamma+\mu) q^{2} \sin ^{2} \theta\right\}
$$

and

$$
M_{(\mathrm{b})}\left(\mathbf{q}, \mathbf{q}^{\prime}, \mathbf{q}^{\prime \prime} ; \text { LA }, \text { TA, TA }\right)=q q^{\prime}\left[(\beta+\lambda)\left(q \cos \theta-q^{\prime}\right)+2(\gamma+\mu)\left(q-q^{\prime} \cos \theta\right) \cos \theta\right] .
$$

Then substituting Eqs. (6) -(8) into Eq. (4) and after a bit of algebra, we obtain for the process (1)

$$
\Gamma^{(1)}=\Gamma_{\mathrm{LA} \rightarrow \mathrm{LA}+\mathrm{TA}}=\frac{\hbar \omega^{5}}{256 \pi \rho^{3}} \frac{\delta^{2}-1}{v ?}(2 \beta+4 \gamma+\lambda+3 \mu)^{2} \int_{x_{0}}^{1} \frac{d x}{x^{2}}\left(1-x^{2}\right)^{2}\left[(1+x)^{2}-\delta^{2}(1-x)^{2}\right]\left[1+x^{2}-\delta^{2}(1-x)^{2}\right]^{2},
$$

where $\delta^{2}=\left(v_{l} / v_{t}\right)^{2}=(\lambda+2 \mu) / \mu$ and $x_{0}=(\delta-1) /(\delta+1)$ with $v_{l}$ and $v_{t}$ being the velocities of the longitudinal and transverse phonons, respectively. ${ }^{13}$ For the process (2) we have

$\Gamma^{(2)}=\Gamma_{\mathrm{LA} \rightarrow \mathrm{TA}+\mathrm{TA}}=\frac{\hbar \omega^{5}}{32 \pi \rho^{3}} \frac{1}{\left(v^{2} v_{t}\right)^{3}} \int_{x_{1}}^{x_{2}}\left\{\left(A+B \delta x-B x^{2}\right)^{2}+\left[C x(\delta-x)-\frac{D}{\delta-x}\left(x-\delta-\frac{1-\delta^{2}}{4 x}\right)\right]^{2}\right\} d x$,

where $x_{1}=(\delta-1) / 2, x_{2}=(\delta+1) / 2$, and

$$
\begin{aligned}
& A=\frac{1}{2}\left(1-\delta^{2}\right)\left[\beta+\lambda+\left(1+\delta^{2}\right)(\gamma+\mu)\right], \\
& B=\beta+\lambda+2 \delta^{2}(\gamma+\mu), \\
& C=\beta+\lambda+2(\gamma+\mu) \\
& D=\left(1-\delta^{2}\right)(2 \beta+4 \gamma+\lambda+3 \mu) .
\end{aligned}
$$

In order to evaluate the magnitude of the decay rate according to Eqs. (9) and (10) we require the numerical values of the elastic constants $\lambda, \mu, \beta$, and $\gamma$. There seem to be some ambiguities in defining these constants from the elastic constants of anisotropic solids. In this work we employ the following procedure to determine those values. First, we denote by $C_{i j k l m n} \ldots$ and $\tilde{C}_{i j k l m n} \ldots$ the elastic constants of a real solid and those of the isotropic model, ${ }^{11}$ respectively. Then the most natural definition of $\tilde{C}_{i j k m n} \ldots$ may be given by minimizing the quantity

$$
f=\sum_{i, j, k, l, m, n, \ldots}\left(\tilde{C}_{i j k l m n} \ldots-C_{i j k l m n} \ldots\right)^{2} .
$$

This is a simple generalization of the scheme adopted by Fedorov ${ }^{14}$ in the definition of the isotropic medium most similar to the given crystal. Applying this scheme, we obtain for cubic crystals

$$
\begin{aligned}
& \lambda=\frac{1}{5}\left(C_{11}+4 C_{12}-2 C_{44}\right), \\
& \mu=\frac{1}{5}\left(C_{11}-C_{12}+3 C_{44}\right), \\
& \alpha=\frac{1}{35}\left(C_{111}+18 C_{112}+16 C_{123}-30 C_{144}-12 C_{166}+16 C_{456}\right), \\
& \beta=\frac{1}{35}\left(C_{111}+4 C_{112}-5 C_{123}+19 C_{144}+2 C_{166}-12 C_{456}\right), \\
& \gamma=\frac{1}{35}\left(C_{111}-3 C_{112}+2 C_{123}-9 C_{144}+9 C_{166}+9 C_{456}\right) .
\end{aligned}
$$

TABLE I. Parameters used in the calculation of decay rates.

\begin{tabular}{llcccccc}
\hline \multicolumn{1}{c}{ Crystals } & \multicolumn{1}{c}{$\gamma$} & \multicolumn{1}{c}{$\begin{array}{c}\lambda \\
\left(10^{11} \mathrm{dyn} / \mathrm{cm}^{2}\right)\end{array}$} & $\mu$ & $\begin{array}{c}v_{l} \\
(\mathrm{~cm} / \mathrm{sec})\end{array}$ & $\begin{array}{c}\rho \\
\left(\mathrm{g} / \mathrm{cm}^{3}\right)\end{array}$ \\
\hline Si- $a$ (Ref. 17) & -4.29 & -9.45 & 5.24 & 6.80 & 9.00 & 5.40 & 2.33 \\
Si- $b$ (Ref. 18) & -5.52 & -8.78 & 5.24 & 6.81 & 9.00 & 5.41 & 2.33 \\
Ge (Ref. 18) & -7.32 & -7.08 & 3.76 & 5.61 & 5.31 & 3.25 & 5.32 \\
GaAs-a (Ref. 19) & -9.73 & -6.71 & 4.30 & 4.86 & 5.13 & 3.02 & 5.32 \\
GaAS-b (Ref. 20) & -5.48 & -6.76 & 4.30 & 4.87 & 5.13 & 3.02 & 5.32 \\
LiF (Ref. 29) & -9.43 & -7.73 & 3.55 & 5.14 & 7.29 & 4.45 & 2.60 \\
NaF (Ref. 30) & -9.47 & -4.43 & 2.73 & 3.10 & 5.66 & 3.34 & 2.79 \\
Fused silica (Ref. 31) & +5.4 & -1.32 & 1.61 & 3.12 & 5.97 & 3.76 & 2.20 \\
\hline \hline
\end{tabular}


The last three equations coincide with the results obtained by an averaging procedure introduced to study the elastic properties of a polycrystalline solid. ${ }^{15}$ We tabulate in Table I the values of isotropically approximated elastic constants for several solids determined by Eq. (13). ${ }^{16}$ For Si and GaAs there exist two sets of data on the second- and third-order elastic constants measured independently by different groups $^{17-20}$ and then we have simultaneously listed the values obtained from each data.

Making use of these values we have computed the decay rates of the LA phonons at $1-\mathrm{THz}$ frequency. Results are given in Table II. It is recognized that the decay into a set of TA phonons [process (2)] dominates the process (1) by a factor of 2-4 for almost all quasi-isotropic samples exemplified in this Rapid Communication, though in fused silica two decay processes have comparable magnitudes. This reflects essentially the difference of the densities of twophonon final states between the processes (1) and (2), ${ }^{21}$ and is rather in accord with the result deduced by Klemens. ${ }^{6}$ We also find that the magnitudes of the decay rates at 1$\mathrm{THz}$ frequency range from $10^{5}$ to $10^{6} \mathrm{sec}^{-1}$. (The small decay rates in $\mathrm{Si}$ are attributed to the smallness of its twophonon density of states.) These values are about one order of magnitude smaller than the decay rates estimated in Refs. 5 and $6 .^{22}$ This suggests that the original works by Orbach and Vredevoe ${ }^{5}$ and Klemens ${ }^{6}$ cannot be applied for a quantitative comparison of the LA-phonon lifetimes with experimental data, mainly because the conclusion of the former is not sound and the latter relies on a simplified Grüneisen approximation. ${ }^{23}$ It should be noted that the decay rate of about $4 \times 10^{7} \mathrm{sec}^{-1}$ for $1-\mathrm{THz}$ LA phonons in GaAs being assumed in several recent papers ${ }^{2,4}$ is again overestimated at least by one order of magnitude. It may be interesting to note that the decay rate of the LA phonons in $\mathrm{CaF}_{2}$ reported by Baumgartner, Engelhardt, and $\mathrm{Renk}^{7}$ indicates $\Gamma=8.2 \times 10^{5} \mathrm{sec}^{-1}$ at $1 \mathrm{THz}{ }^{24}$ This value is also within the range of the decay rates given in Table II.

For comparison's sake the isotope scattering rates of 1$\mathrm{THz}$ phonons are also given in Table II. ${ }^{25}$ (Dispersive effects are neglected.) We observe that at frequencies lower than $1 \mathrm{THz}$ the probability of the spontaneous decay of the LA phonons is much smaller than that of the scattering by isotopic atoms in isotopically impure crystals.

The decay rates in Table II have been obtained on the basis of the room-temperature values of elastic constants. It
TABLE II. Calculated decay rates of $1-\mathrm{THz}$ LA phonons together with the scattering rates by isotopic atoms.

\begin{tabular}{|c|c|c|c|c|}
\hline Crystals & $\underset{\left(\sec ^{-1}\right)}{\Gamma}$ & $\Gamma^{(1)} / \Gamma$ & $\Gamma^{(2)} / \Gamma$ & $\begin{array}{l}\Gamma_{\text {isotope }} \\
\left(\sec ^{-1}\right)\end{array}$ \\
\hline $\mathrm{Si}-a$ & $7.41 \times 10^{4}$ & 0.204 & 0.796 & \\
\hline $\mathrm{Si}-b$ & $7.25 \times 10^{4}$ & 0.204 & 0.796 & \\
\hline $\mathrm{Ge}$ & $6.43 \times 10^{5}$ & 0.260 & 0.740 & $3.67 \times 10^{7}$ \\
\hline GaAs- $a$ & $1.35 \times 10^{6}$ & 0.263 & 0.737 & $738 \times 10^{6}$ \\
\hline $\mathrm{GaAs}-b$ & $7.72 \times 10^{5}$ & 0.222 & 0.778 & \\
\hline $\mathrm{LiF}$ & $5.14 \times 10^{5}$ & 0.330 & 0.670 & $1.69 \times 10^{6}$ \\
\hline $\mathrm{NaF}$ & $2.80 \times 10^{6}$ & 0.292 & 0.708 & 0 \\
\hline Fused silica & $7.99 \times 10^{5}$ & 0.540 & 0.460 & $3.85 \times 10^{6}$ \\
\hline
\end{tabular}

has been shown that the elastic constants depend to some degree on temperatures. ${ }^{26}$ Only for $\mathrm{Si}$ and Ge the temperature dependence of the third-order elastic constants has been measured. ${ }^{27}$ In terms of the data at $3 \mathrm{~K}$ we have also calculated the decay rates and obtained $1.23 \times 10^{5}$ and $1.62 \times 10^{6} \mathrm{sec}^{-1}$ for $1-\mathrm{THz} \mathrm{LA}$ phonons in $\mathrm{Si}$ and $\mathrm{Ge}$, respectively. These values are larger than those at room temperature by factors of 1.7-2.5. The same kinds of results will be also expected for the decay rates in GaAs, $\mathrm{LiF}, \mathrm{NaF}$, and fused silica at low temperatures. However, the branching ratios do not essentially change with temperatures and our conclusions on the spontaneous decay of the LA phonons are still valid at low temperatures, though more elaborate, systematic measurements of third-order elastic constants down to liquid-helium temperatures are desired.

To summarize, we have evaluated the spontaneous decay rate of the LA phonons in the isotropic model in terms of the second- and third-order elastic constants. Our numerical results show that the dominant decay channel is $\mathrm{LA} \rightarrow \mathrm{TA}+\mathrm{TA}$ and the total decay rate ranges from $10^{5}$ to $10^{6} \mathrm{sec}^{-1}$ at $1 \mathrm{THz}$, indicating that the currently assumed down-conversion rates of the LA phonons ${ }^{2,4,8,28}$ are correct in neither their branching ratios nor absolute magnitudes.

The author would like to thank Professor T. Sakuma for a critical reading of the manuscript. This work is partially supported by a Grant in Aid for Scientific Research from the Ministry of Education, Science and Culture, Japan.
${ }^{1}$ D. V. Kazakovtsev and Y. B. Levinson, Pis'ma Zh. Eksp. Teor. Fiz. 27, 194 (1978) [JETP Lett. 27, 181 (1978)]; Phys. Status Solidi B 96, 117 (1979).

${ }^{2}$ N. N. Guseinov and Y. B. Levinson, Solid State Commun. 45, 371 (1983); Zh. Eksp. Teor. Fiz. 85, 779 (1983) [Sov. Phys. JETP 58, $452(1983)]$.

${ }^{3}$ M. Lax, V. Narayanamurti, P. Hu, and W. Weber, J. Phys. (Paris) Colloq. 42, C6-161 (1981).

${ }^{4}$ M. Lax, V. Narayanamurti, R. Ulbrich, and N. Holzarth, in Phonon Scattering in Condensed Matter, edited by W. Eisenmenger, K. Lassman, and S. Dottinger (Springer, Berlin, 1984), p. 103.

${ }^{5}$ R. Orbach and L. A. Vredevoe, Physics (N.Y.) 1, 91 (1964).

6P. G. Klemens, J. Appl. Phys. 38, 4573 (1967).

${ }^{7}$ R. Baumgartner, M. Engelhardt, and K. F. Renk, Phys. Rev. Lett. 47, 1403 (1981).

${ }^{8}$ R. S. Markiewicz, Phys. Rev. B 10, 4674 (1980).

9P. F. Tua and G. D. Mahan, Phys. Rev. B 26, 2208 (1982).
10J. W. Tucker and V. W. Rampton, Microwave Ultrasonics in Solid State Physics (North-Holland, Amsterdam, 1972), Chap. 4.

${ }^{11}$ S. Tamura, Phys. Rev. B 30, 610 (1984).

${ }^{12}$ At frequencies around $1 \mathrm{THz}$, dispersive effects are more important than the effects of energy broadening due to scatterings.

${ }^{13}$ Comparing Eq. (9) with Eq. (3) of Ref. 5, we find that the result of Orbach and Vredevoe for the decay rate of the process (1) is not exact in the isotropic model but may be derived with some additional approximations. In our notation their result is

$$
\Gamma_{\mathrm{OV}}^{(1)}=\frac{\hbar \omega^{5}}{16 \pi \rho^{3}} \frac{\delta^{2}-1}{v ?}(2 \beta+4 \gamma+\lambda+3 \mu)^{2}\left(\frac{3}{8} \delta-1+\frac{1}{\delta}\right) \text {. }
$$

${ }^{14} \mathrm{~F}$. I. Fedorov, Theory of Elastic Waves in Crystals (Plenum, New York, 1968), Chap. 5.

${ }^{15}$ R. A. H. Hamilton and J. E. Parott, J. Phys. C 1, 829 (1968).

${ }^{16}$ The values for the original elastic constants of both the second 
and third orders used in the calculations are those measured at room temperatures.

17J. J. Hall, Phys. Rev. 161, 756 (1967).

${ }^{18}$ H. J. McSkimin and P. Andreatch, Jr., J. Appl. Phys. 35, 3312 (1964).

19J. R. Drabble and A. J. Brammer, Solid State Commun. 4, 467 (1966).

${ }^{20}$ H. J. McSkimin and P. Andreatch, Jr., J. Appl. Phys. 38, 2610 (1967).

${ }^{21}$ Fused silica is unusual in the fact that most of third-order elastic constants are positive.

${ }^{22}$ For a typical solid Orbach and Vredevoe (Ref. 5) have estimated the decay rate to be $2 \times 10^{7} \mathrm{sec}^{-1}$ at $1 \mathrm{THz}$ and Klemens's formula (Ref. 6) predicts $8 \times 10^{6} \mathrm{sec}^{-1}$ for a solid with the Grüneisen constant of 2 .

${ }^{23}$ With the use of the formula by Orbach and Vredevoe and numerical values for GaAs- $a$ (upper row) in Table I, we have obtained $\Gamma_{\mathrm{OV}}^{(1)}=1.78 \times 10^{6} \mathrm{sec}^{-1}$ which is still about a factor of 5 larger than $\Gamma^{(1)}=3.69 \times 10^{5} \mathrm{sec}^{-1}$ obtained by our calculations.
${ }^{24} \mathrm{As}$ far as we know the third-order elastic constants have not been measured for $\mathrm{CaF}_{2}$.

${ }^{25}$ See, for example, S. Tamura, Phys. Rev. B 30, 849 (1984). There is a typographical error in Eq. (10) of this paper. The correct expression is

$$
\tilde{g}=\sum_{\sigma} g(\sigma) M^{2}(\sigma) /\left(\sum_{\sigma} M(\sigma)\right)^{2} .
$$

${ }^{26}$ See, for example, H. J. McSkimin, J. Appl. Phys. 24, 988 (1953).

27J. Philip and M. A. Breazeale, J. Appl. Phys. 54, 752 (1983). In their data $C_{144}$ depends very sensitively on temperature and its room temperature values deviate considerably from those measured by McSkimin and Andreatch (Ref. 18) in both Si and Ge.

${ }^{28}$ W. E. Bron, Phys. Rev. B 21, 2627 (1980).

29 J. R. Drabble and R. E. B. Strathen, Proc. Phys. Soc. London 92, 1090 (1967).

30W. A. Bensch, Phys. Rev. B 6, 1504 (1972).

${ }^{31}$ W. T. Yost and M. A. Breazeale, J. Appl. Phys. 44, 1909 (1973). 